\title{
Differential expression of the CCN family member WISP-1, WISP-2 and WISP-3 in human colorectal cancer and the prognostic implications
}

\author{
SIMON R. DAVIES, MANSEL LEIGH DAVIES, ANDREW SANDERS, \\ CHRIS PARR, JARED TORKINGTON and WEN G. JIANG \\ Metastasis and Angiogenesis Research Group, Cardiff University School of Medicine, \\ Heath Park, CF14 4XN Cardiff, UK
}

Received December 8, 2009; Accepted January 25, 2010

DOI: 10.3892/ijo_00000595

\begin{abstract}
The WISPs (Wnt-inducted secreted proteins, WISP-1, WISP-2 and WISP-3) are part of the CCN family. These molecules are known to play a diverse role in cells but their role in cancer cells remains controversial. We analysed the expression of the three WISP molecules at the mRNA and protein levels in a cohort of 94 human colorectal tumours and 80 normal colorectal tissues and correlated the results with the pathological features and clinical outcome of the patients. WISP-1 transcripts were found at higher levels in the tumour samples than in the normal tissue $(p=0.0015)$; higher in patients with Dukes stage B and C compared to Dukes A ( $p=0.017$ and $p=0.024$, respectively); higher in patients with moderately and poorly differentiated cancers compared to the well differentiated cancers $(\mathrm{p}=0.020$ and $\mathrm{p}=0.076$, respectively and $\mathrm{p}=0.0035$ when combined); higher in node positive tumours compared with the node negative $(\mathrm{p}=0.11)$ and in the patients with higher TNM staging (TNM 2, 3 and 4 compared to TNM $1 \mathrm{p}=0.037$ ). WISP-2 showed the opposite pattern with lower levels of expression in cancer cells compared to normal $(\mathrm{p}=0.082)$. Although no significant differences were found within the cancer group when indices of a more aggressive tumour were compared to the normal tissue a significant reduction in expression was found (Dukes $\mathrm{C} p=0.044$, poorly differentiated $\mathrm{p}=0.019$, TNM $3 \mathrm{p}=0.020$ and node positive disease $\mathrm{p}=0.048$ ). WISP-3 transcript levels showed no significant differences between groups. WISPs may play important but contrasting roles in colorectal cancer with WISP-1 appearing to act as a factor stimulating aggressiveness, WISP-2 as a tumour suppressor and WISP-3 having no definable beneficial or detrimental role.
\end{abstract}

Correspondence to: Dr Wen G. Jiang, Metastasis and Angiogenesis Research Group, Department of Surgery, Cardiff University School of Medicine, Heath Park, CF14 4XN Cardiff, UK E-mail: jiangw@cf.ac.uk

Key words: WISP-1, WISP-2, WISP-3, CCN, colorectal cancer

\section{Introduction}

The CNN family comprises a growing number of molecules. The first to be discovered where cysteine-rich 61 (Cyr61/ CCN1), connective tissue growth factor (CTGF/CCN2) and nephroblastoma over-expressed (Nov/CCN3) (1). The next to be identified were three Wnt-inducted secreted proteins which were named WISP-1/CCN4, WISP-2/CCN5 and WISP-3/CCN6.

Upstream of the CCN family is Wnt-1, a cycleine-rich glycosylated signalling protein, that was originally identifies as an oncogene activated by the insertion of mouse mammary tumour virus in virus induced mammary adenocarcinoma (2-4). Although Wnt-1 has been shown to produce a significant tumor reaction in mice the evidence of its involvement in human cancer has not been so clearly defined. Wnt-1 is just one of 19 Wnt proteins that are important regulators in the signalling pathway. This pathway has diverse roles in governing cell fate, proliferation, migration, polarity and death. The central Wnt signalling pathway is activated by the engagement of some of the Wnt proteins to the seventransmembrane spanning Frizzled receptors and recruiting the cytoplasmic protein Dishevelled (Dsh) to the cell membrane. Dsh inhibits kinase activity of active glycogen synthase kinase-3ß (GSK-3ß) resulting in an increase in B-catenin levels. $\beta$-catenin has been associated with colonic carcinomas and melanomas implicating the Wnt pathway in tumourigenesis.

It has been shown that three different genes are up-regulated in Wnt-1 transformed cells WISP-1, WISP-2 and the related gene WISP-3, all members of the CCN family of growth factors (5). The CCN family as a whole appear to stimulate mitosis, adhesion, apoptosis, extracellular matrix production and migration and growth arrest (6). These functions are probably due to the ability of the family to bind and activate cell-surface integrins and intracellular signalling molecules including fibulin 1C, Notch 1, S100A4 and ion channels (7). Despite these advances in understanding the biology of the molecules in cells and their signalling pathways their role in cancer is unclear and no definitive results have been reported. The WISPs have previously been implicated as 
being significant in tumourigenesis and metaplasia in a variety of tissues. The effects are not however universal with the proteins exerting transforming and growth stimulatory effects on some cell types while causing inhibition of growth and metastasis of tumour in others.

The WISP-1 gene, which is located on the 8q24.1-8q24.3, shows this variation of action. It has been shown that there is an overexpression of WISP-1 RNA in colon cancer cell lines compared to normal mucosa $(5,8)$ and it was concluded that WISP-1 may play a role in tumourigenesis, possibly by promoting cell cycle checkpoint progression, accelerating cell growth and inhibiting apoptosis. By contrast, the over expression of WISP-1 in H460 lung cancer cells was found to lead to an inhibition of lung metastasis and in vitro cell invasion and motility (9). WISP-1 also protects cells from $\mathrm{p} 53-$ mediated apoptosis in response to DNA damage through the activation of Akt kinase, up-regulation of $\mathrm{Bcl}-\mathrm{X}_{\mathrm{L}}$, and inhibition of cytochrome $c$ release (10).

The WISP-2 gene, located on 20q12-20q13, has like the other members of the $\mathrm{CCN}$ family, been implicated as having an important role in both angiogenesis and carcinogenesis (6). In contrast to WISP-1, WISP-2 DNA was amplified but its mRNA expression was significantly reduced in the majority of tumours compared to normal colonic mucosa suggesting it may act as a tumour suppressor (5). This is in stark contrast to the actions of WISP-2 in human breast disease. The mRNA and protein levels are elevated in tumour-derived cell lines but the levels were barely detectable in normal breast epithelial cells. Silencing the WISP-2 gene functions also minimizes serum-induced breast tumour cell proliferation (11) but by contrast it can be up-regulated in non-invasive MCF-7 human breast tumour cells by epithelial growth factor (EGF) which is believed to be linked to poor prognosis in breast cancer (12).

The WISP-3 gene is located on 6q22-6q23. As seen with WISP-1 the overexpression of WISP-3 has been linked to colonic tumours $(5,13)$. Previous studies concluded that overexpression was associated with the development of colonic tumours. Where WISP-3 differs from WISP-1 is in the fact that there is an overexpression of WISP-3 RNA without DNA amplification. Interesting the effects of WISP-3 in inflammatory breast cancer appears to play an important role as a tumour suppressor gene, again the reverse of the previous finding for colon cancer. WISP-3 has also been linked to connective tissue disease progression in conditions such as pseudorheumatoid dysplasia and polyjuvenile idiopathic arthritis. It regulates the expression of cartilagespecific molecules that sustain chondrocyte growth and cartilage integrity by regulating collagen II, aggrecan and possibly promote superoxide dismutase expression in the condrocysts (14).

The role of the WISP family in the development of clinical cancer is unclear and in some cases controversial and unproven. In the present study we examine the relationship between each of the three Wnt-inducted secreted proteins looking at their levels in a cohort of human colorectal cancer and studied the clinical relevance. Until now these proteins have not been studied together and directly compared in human colorectal tumours.

\section{Materials and methods}

Sample collection. Colorectal cancer tissue $(n=94)$ and normal background tissue $(n=80)$ were collected immediately after surgery and stored in a deep freeze until use. The presence of tumour cells in the collection tissues was verified by a consultant pathologist, using hematoxylin and eosin (H\&E) stained frozen sections $(15,16)$. Details of the histology were obtained from pathology reports and together with basic patient demographics are shown in Table I. The study was approved by the local ethic committee and consent was obtained from the patients.

Materials. RNA-extraction kits and RT kits were obtained from AbGene, Guildford, Surrey, UK. PCR primers were designed using Beacon Designer (Palo Alto, CA, USA) and synthesized by Invitrogen (Paisley, Scotland, UK). Molecular-biologygrade agarose and DNA ladder were sourced from Invtrogen while the Mastermix for routine PCR and quantitative PCR came from AbGene (Surrey, UK). WISP-1, 2 and 3 antibodies were purchased from Santa Cruz Biotechnologies Inc. (Santa Cruz, CA, USA. WISP-1 (sc-8865) a goat polyclonal antibody raised against a peptide mapping at the carboxy terminus of WISP-1 of human origin; WISP-2 (sc-8868) goat polyclonal antibody mapping the C-terminus of WISP-2 of human origin; and WISP-3 (sc-25443) rabbit polyclonal antibody against amino acids 231-295 of WISP-3 of human origin). Anti-actin was also from Santa Cruz Biotechnologies Inc.

Tissue procession, RNA extraction and cDNA synthesis. Frozen sections of tissue were cut to thicknesses of 5-10 $\mu \mathrm{m}$ and kept for immunohistochemistry and routine histology (17). A further 15-20 sections were homogenized using a hand held device in ice-cold RNA extraction solution. The concentration of the RNA was determined using a UV spectrophotometer. Using an RT kit with an anchored oligo-dT primer (supplied by AbGene) reverse transcription was carried out using $1 \mu \mathrm{g}$ total RNA in a 96-well plate. The quality of the cDNA was verified using $B$-actin primers.

Quantitative analysis of WISP family members. The level of mRNA expression of WISP-1-3 from the above prepared CDNA was determined by real-time quantitative PCR, based on the Amplifluor ${ }^{\mathrm{TM}}$ technology (18), modified from a previously reported method $(17,19,20)$. A pair of PCR primers was designed using Beacon Designer software (version 2). To one of the primers (an antisense primer routinely used in our laboratory) an additional sequence, known as the $\mathrm{Z}$ sequence (5'-ACTGAACCTGACCGTACA-3') which is complementary to the universal $\mathrm{Z}$ probe (18) (Intergen, Oxford, UK), was added. Cytokeratin-19 (CK19) was used for comparison of cellularity during the analysis. The primers used are given in Table II.

The reaction was performed using: Hotstart Q-master mix (Abgene), $10 \mathrm{pmol}$ of specific forward primer, 1 pmol of reverse primer which has the $\mathrm{Z}$ sequence, $10 \mathrm{pmol}$ of 6-carboxyfluorescein (FAM)-tagged probe (Intergen, Oxford, UK), and cDNA from approximately $50 \mathrm{ng}$ RNA (calculated from the starting RNA in the reverse transcriptase reaction). The reaction was carried out using IcyclerIQ ${ }^{\mathrm{TM}}$ (Bio-Rad, 
Table I. Clinical and pathological information on the study cohort.

\begin{tabular}{lcc}
\hline Clinical information & Number (n) & Percentage (\%) \\
\hline Dukes stage & & \\
A & 15 & 19.5 \\
B & 32 & 41.6 \\
C & 30 & 39.0 \\
T stage & & \\
T1 & 8 & 10.4 \\
T2 & 10 & 13 \\
T3 & 40 & 51.9 \\
T4 & 19 & 24.7 \\
Differentiation & & \\
Well differentiated & 10 & 13.0 \\
Moderately differentiated & 53 & 68.8 \\
Poorly differentiated & 14 & 18.2 \\
Nodal status & & \\
Negative & 45 & 58.4 \\
Positive & 32 & 41.6 \\
Sex & 45 & \\
Male & & \\
Female & & \\
\hline
\end{tabular}

Hemel Hempstead, UK) which is equipped with an optic unit that allows real time detection of the 96 reactions under the following conditions: $94^{\circ} \mathrm{C}$ for $5 \mathrm{~min}, 50$ cycles of: $94^{\circ} \mathrm{C}$ for $15 \mathrm{sec}, 55^{\circ} \mathrm{C}$ for $35 \mathrm{sec}$ and $72^{\circ} \mathrm{C}$ for $20 \mathrm{sec}(19,21-23)$. The levels of the transcripts were generated using an internal standard (17) that was simultaneously amplified with the samples, and are shown here in two ways: levels of transcripts based on equal amounts of RNA, and as a target/CK19 ratio.

Immunohistochemical staining of the WISP family proteins. The frozen sections of colorectal tumour and background tissue were cut to a thickness of $6 \mu \mathrm{m}$ using a cryostat (23). The samples were mounted onto Super Frost Plus microscope slides, air-dried and then fixed in a mixture of $50 \%$ acetone and $50 \%$ methanol and air dried once again. Endogenous peroxydise was block by submerging the slides in pure ethanol $/ \mathrm{H}_{2} \mathrm{O}_{2}$ for $15 \mathrm{~min}$. The sections were then placed into Optimax wash buffer for $20 \mathrm{~min}$ in a $0.6 \%$ horse serum blocking solution and probed with the primary antibody. Extensive washing was carried out before the sections were incubated with the secondary biotinylated antibody (Multilink Swine anti-goat/mouse/rabbit immunoglobulin made up in wash buffer and horse serum to manufacturer's specification; Vector Labs, UK) for $30 \mathrm{~min}$. Following further washing, avidin-biotin complex (Vector Latoratories, Burlingame, CA, USA) was then applied before a final washing of the samples. Diaminobenzidine chromogen (Vector Lab) was then added to the sections which were then incubated in the dark for 5 min before being counter stained in Gill's haematoxylin and dehydrated in ascending grades of methanol before cleaning with xylene and mounting under a cover slip.

Statistical analysis. The statistical analysis was carried out using the t-test (Minitab version 12.1).

\section{Results}

Expression of WISP members in colorectal tissue. Using quantitative PCR it can be seen that WISP-1 displayed a higher level in tumour tissues compared to normal tissue, statistically significant at $\mathrm{p}=0.0015$ (Fig. 1 left hand panel). WISP-2 displays lower levels of transcripts, just below significance levels $\mathrm{p}=0.082$ (Fig. 1 middle panel) while WISP-3, like WISP-1 is present in greater volumes (Fig. 1 right hand panel), however, not to a significant level $\mathrm{p}=0.86$.

Distribution of WISP members in different cell types. On immunohistochemical analysis (Fig. 2) WISP-1 showed strong staining of the normal epithelial cells, which was confined mostly to the cell membrane areas with slight staining of stromal tissue. In carcinoma tissues the staining for WISP-1 was overall found to be more intense with an increase in cytoplasmic staining and a decrease in the staining around the cell membrane areas, again there was slight or no stromal staining. In contrast WISP-2 (Fig. 2 middle panel) strongly stained in the cell membrane areas as for WISP-1, but in the carcinoma tissues there was reduced overall staining with very slight cell membrane or cytoplasmic staining. For WISP-3 (Fig. 2 bottom) the staining pattern in normal tissues was of a similar pattern as found in normal tissues stained for WISP-1 and 2, however, in carcinoma tissues WISP-3 demonstrated more generalised cytoplasmic staining particularly in the basal areas of the tissue again with less cell membrane area staining and slight or no stromal staining.

Table II. Primer sequences for quantitative PCR.

\begin{tabular}{lll}
\hline & \multicolumn{1}{c}{ Forward primer } & Reverse primer (Z sequence underlined) \\
\hline WISP-1 & CAAGAGGCCACGCAAGAC & ACTGAACCTGACCGTACAGTAGGCTATGCAGTTCCTGT \\
WISP-2 & AGTGGGGCTGGAAGGTCT & $\underline{\text { ACTGAACCTGACCGTACACTCTTGGCAGAGGACGAC }}$ \\
WISP-3 & ACAAAACAAATGCCAGCTTAT & $\underline{\text { ACTGAACCTGACCGTACACATTGGTCACCCTGTTAGAT }}$ \\
CK19 & CAGGTCCGAGGTTACTGAC & $\underline{\text { ACTGAACCTGACCGTACACACTTTCTGCCAGTGTGTCTTC }}$ \\
\hline
\end{tabular}



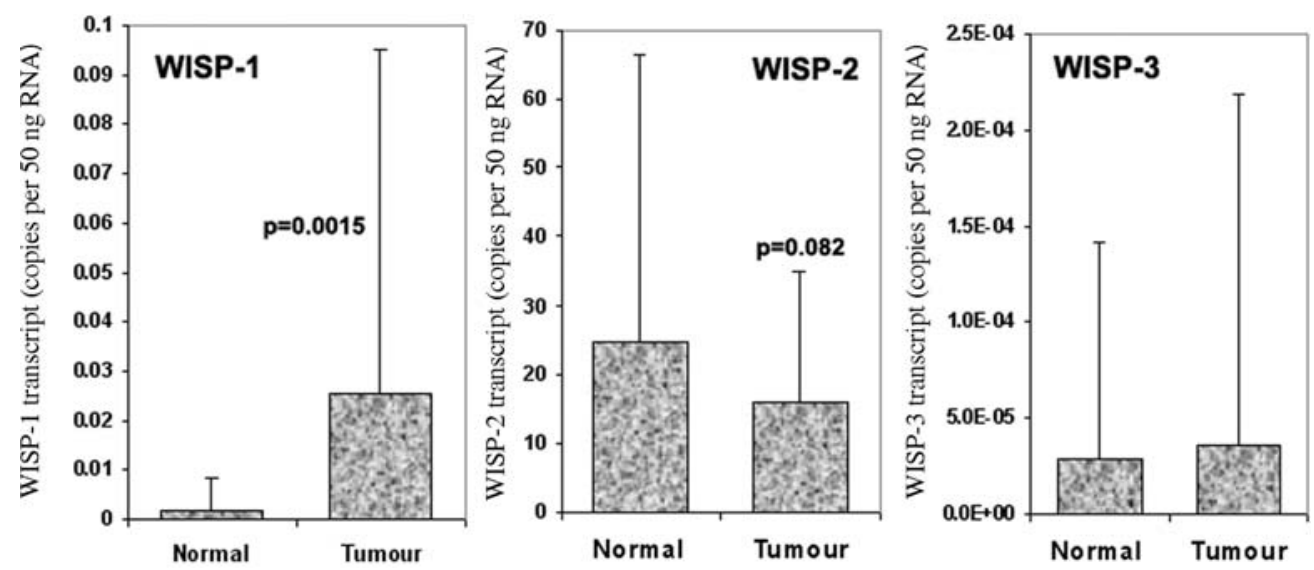

Figure 1. The level of WISP expression in human colorectal tumours compared to normal colorectal tissue.
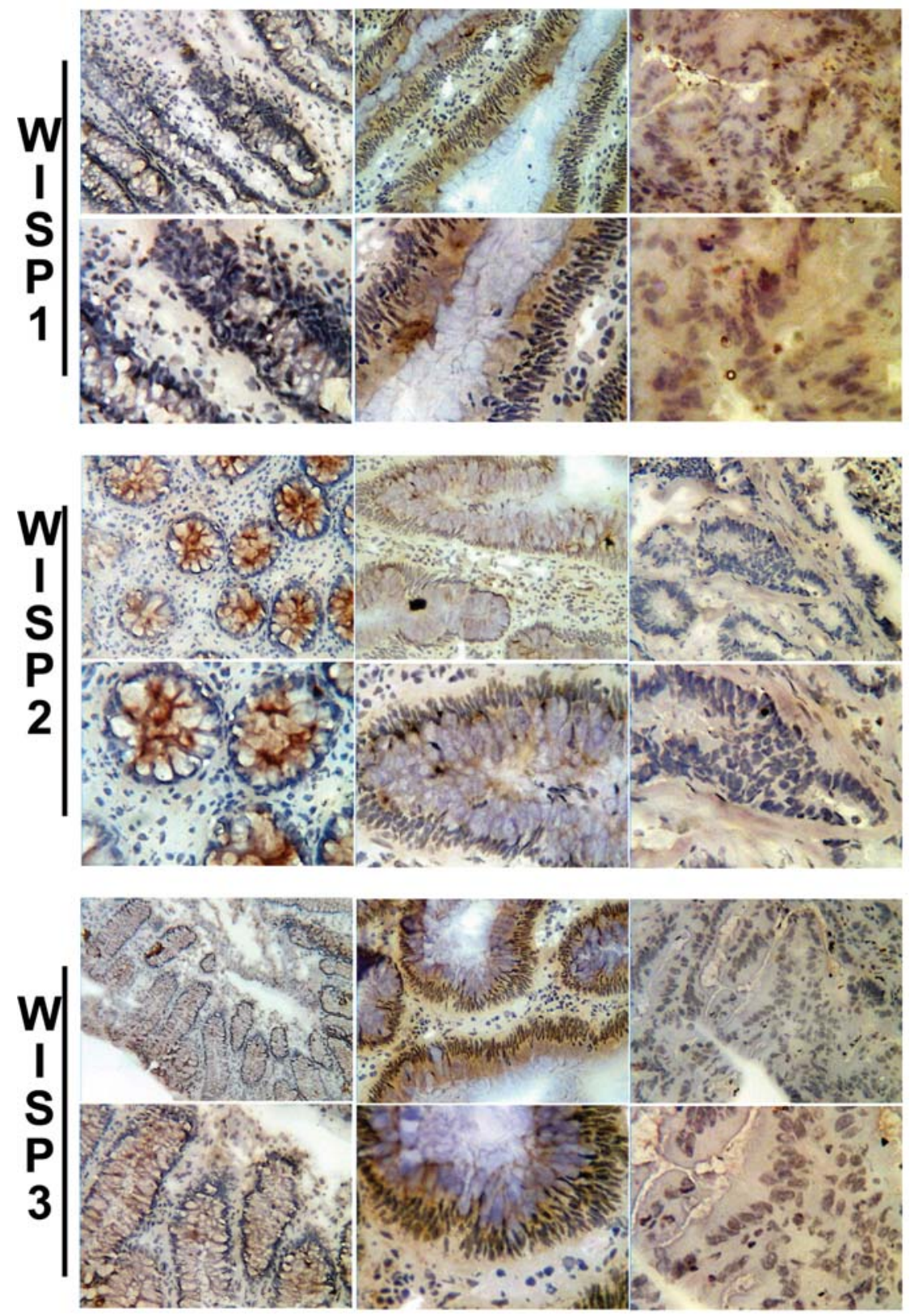

Figure 2. Detection of WISP 1 (top), WISP-2 (middle) and WISP-3 (bottom panel) proteins in colorectal cancer cell lines using immunohistochemical analysis. Left, normal colon tissues; middle, well differentiated carcinoma; right, Dukes B, T-4, TNM-2 tumours. 

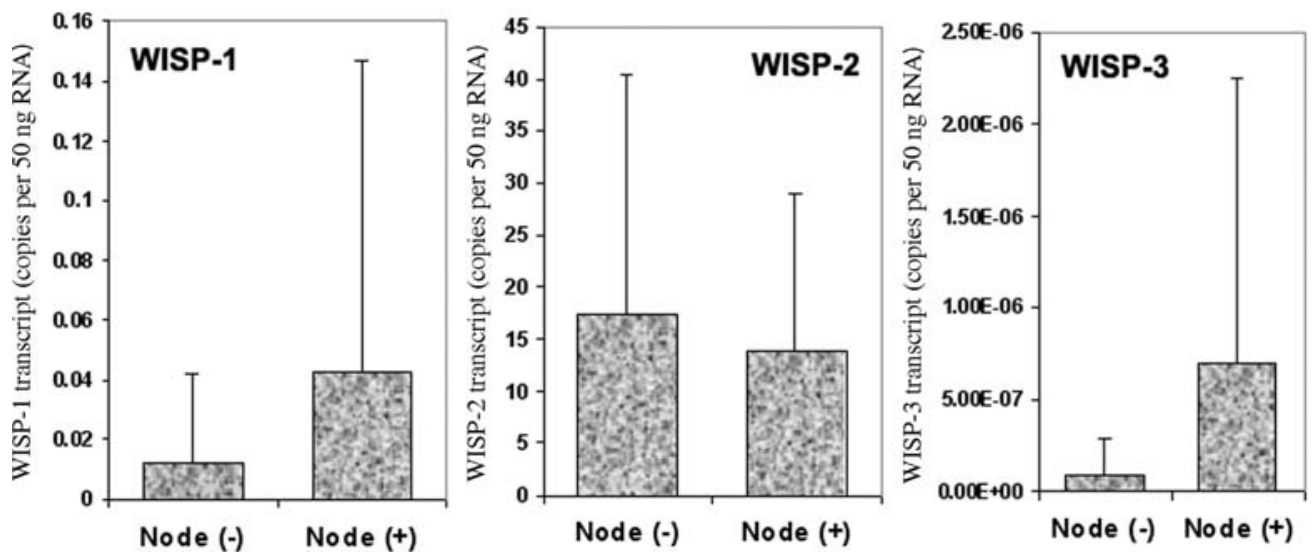

Figure 3. The level of WISP expression when comparing samples from patients with or without nodal involvement.
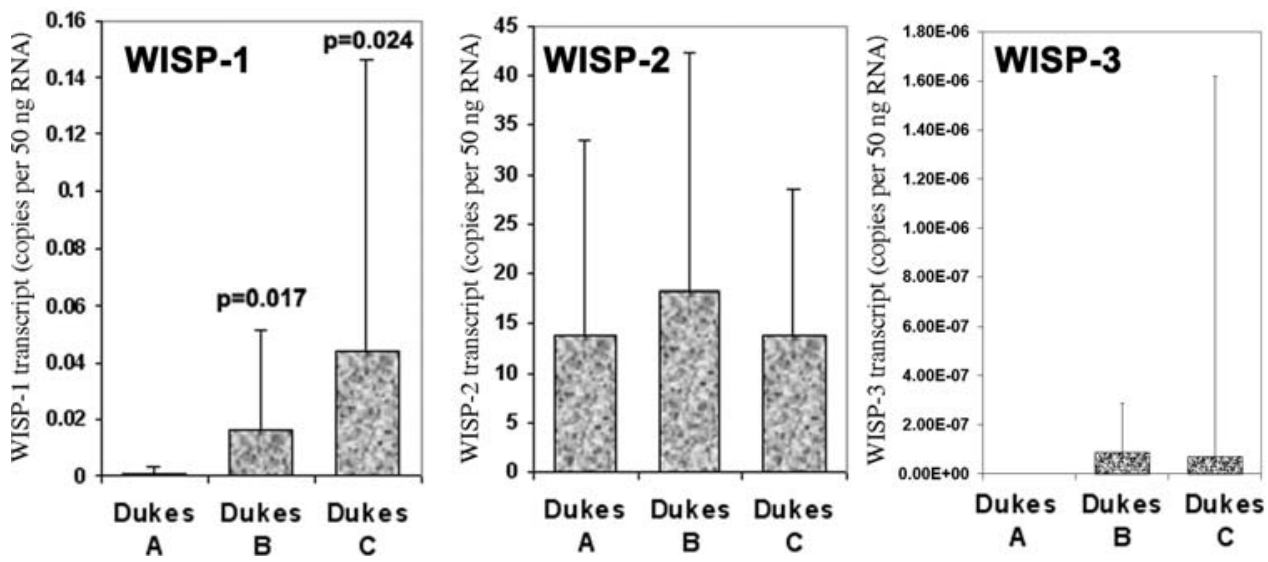

Figure 4. The level of WISP expression when comparing samples from patients with different Dukes staging of their colorectal cancer.

Levels of WISP members and relationship with prognostic indices and nodal involvement. The prognostic indices used in this study are 2-fold; the nodal status and the Dukes classification.

Figs. 3 and 4 show the results. From Fig. 3 it can be seen that there are greater levels of WISP-1 expression in the node positive tumours compared with the node negative $(\mathrm{p}=0.11$, Fig. 3 left hand panel). The reverse is true for WISP-2 in which there are lower levels of expression in the node positive tumours ( $p=0.43$, Fig. 3 middle panel). WISP- 3 again has greater levels in node positive tumours $(p=0.27$ Fig. 3 right hand panel). There is no significant difference, however, when the node positive tumours are compared to the normal tissues, significant differences are found in WISP-1 levels $(\mathrm{p}=0.034)$ and WISP-2 levels $(\mathrm{p}=0.048)$.

When comparing the WISP samples we looked at the differences between levels of the transcript in the different stages of the Dukes classification. The samples were compared to both normal tissue and between the different stages. WISP-1 had significantly different levels in both analyses with all 3 stages having significantly greater levels compared to the normal tissue and when compared to each other the higher stages had the significantly greater levels (Dukes A compared to B p=0.017, A to $\mathrm{C} p=0.024, \mathrm{~A}$ to $\mathrm{B}+\mathrm{C}$ combined $\mathrm{p}=0.0035$ and $\mathrm{B}$ to $\mathrm{C} \mathrm{p}=0.157$, Fig. 4 , left hand panel). In contrast, WISP-2 showed lower levels compared to the normal tissue and no inter-grade differences (Fig. 4, middle panel). WISP-3 transcripts were only identified in a few of the tumour cells and as a result differentiating between the different groups is difficult and no significant differences were found (Fig. 4, right hand panel).

Levels of WISP members and relationship with tumour differentiation and the TNM staging. WISP-1 transcript was found in higher levels in moderately and poorly differentiated tumours compared to well differentiated tumours $(\mathrm{p}=0.020$ and $\mathrm{p}=0.076$, respectively and $\mathrm{p}=0.0035$ when moderately and poorly differentiated tumours were compared together, Fig. 5 left hand panel). WISP-2 showed no significant differences between the differentiation groups, but when they were compared to the normal samples the poorly differentiated patients had a significantly lower level ( $p=0.019$, Fig. 5 middle panel). The numbers of samples expressing WISP-3 was very small and no significant differences were found in the limited results (Fig. 5, right hand panel).

When looking at the TNM staging the trends show higher levels for WISP-1 in the higher TNM stages. The differences were not significant until the transcript levels in TNM 1 staged 

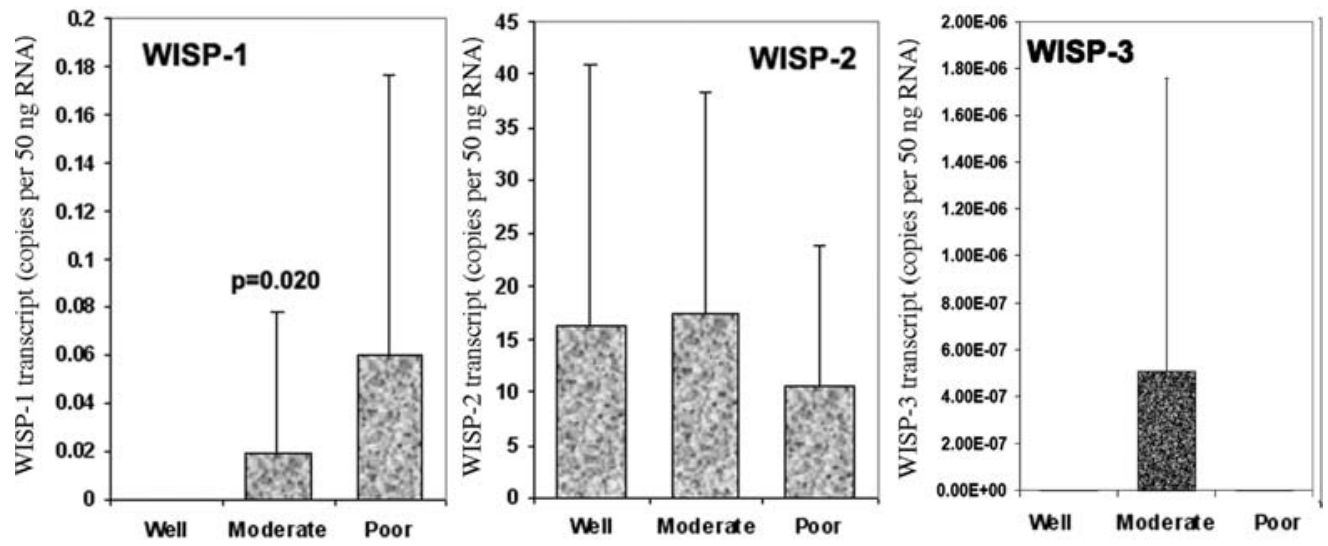

Figure 5. The level of WISP expression when comparing samples from patients with different grades of tumour differentiation.
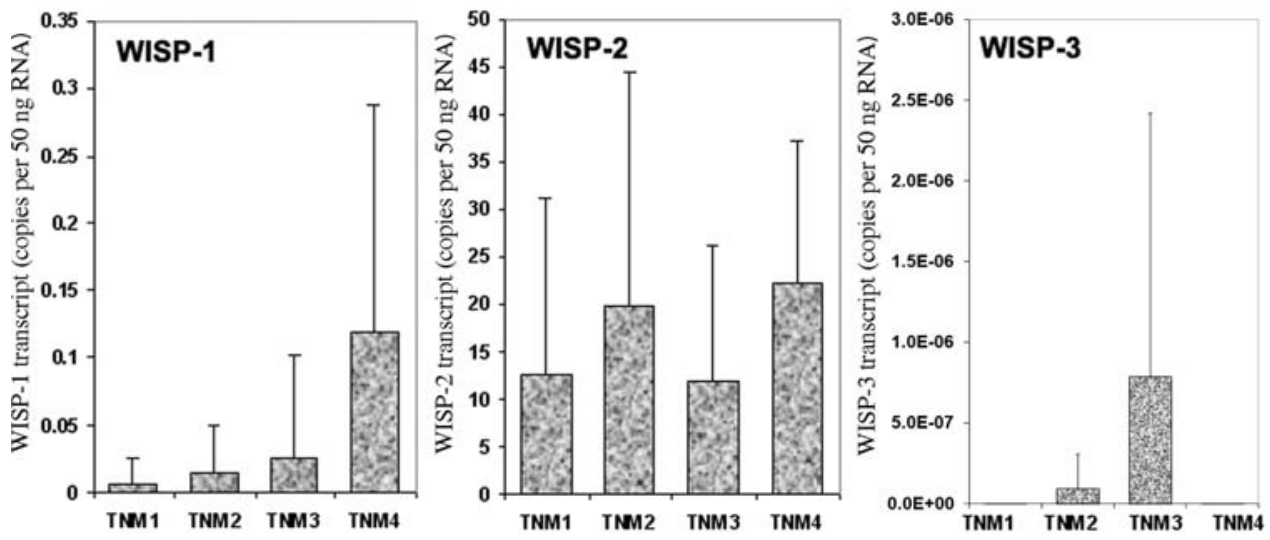

Figure 6. The level of WISP expression when comparing samples from patients with different tumour, node and metastasis (TNM) scores.

patients were compared to the other 3 groups and a significantly higher level was found in more diffuse disease $(\mathrm{p}=0.037$, Fig. 6 left hand panel). There were no significant differences with WISP-2 although the levels were lower in all groups than the normal tissues (Fig. 6 middle panel) and WISP-3 again had little data to allow significant conclusions to be drawn (Fig. 6, right hand panel).

\section{Discussion}

This study shows an aberrant expression of the CCN family member WISP-1, 2 and 3 in human colorectal cancer. The results showed that there are significant clinical implications for the patients depending on which of the WISP transcripts are present.

This study follows on from a similar study looking at the WISP molecules in breast cancer (25). That report concluded that in breast cancer WISP-1 appears to act as a tumour suppressor with transcript levels being inversely proportional to indices of poor prognosis (positive nodal status, higher Nottingham Prognostic Index, higher grade and poor clinical outcome). WISP-2 by contrast was found to be associated with aggressive tumour types with high levels found in patients with the poorest outlook. WISP-3 showed no relationship with prognostic indicators. The purpose of this study was to investigate the role of the WISP molecules in colorectal cancer to see if a similar expression pattern exists.

There were fewer transcripts of WISP-1 and WISP-3 in tumour and normal tissue than WISP-2, however when comparing the transcript levels to normal tissues WISP-1 and to a lesser extent WISP-3 significantly higher transcript levels were found in tumour compared to normal tissue which contrasted with WISP-2 which had lower levels of transcripts.

The clinical significance of this high level of expression of WISP-1 is most clearly seen when comparing the levels for different Dukes grade for colorectal cancer (Fig. 4, right hand panels). It can be seen that with increasing grade the level of transcript significantly increases. This has clinical implications as it has been widely reported that increasing Dukes stage is inversely proportional to survival (26). Other significant differences which have a clinical significance are seen when comparing the differentiation of the tumour (Fig. 5, right hand panel), here again we see that increased WISP-1 levels are found in moderately and poorly differentiated tumours when compared to well differentiated tumours. Poorly differentiated tumours have been shown to have a more aggressive growth pattern with clinical implications. We can conclude that WISP-1 is associated with more aggressive colorectal cancers. The findings of increasing expression are consistent with the early work undertaken by Pennica et al 
(5) that showed RNA overexpression of WISP-1 in human colon tumours compared to normal mucosa. The same finding were also reported by Khor et al (8) who found increased immunohistochemical staining of WISP-1 in colorectal cancers compared to normal tissues. In stark contrast to Khor et al who stated that WISP-1 was associated with well differentiated colorectal tumours we report the opposite. The study by Khor et al was conducted on a relatively small cohort $(n=47)$ and did not look at other aspects of the cancer pathology. The results are in contrast to this department's previous findings into WISP-1 expression in breast cancer. It was found that WISP-1 acts as a tumour suppressor with transcript levels being inversely proportional to tumour aggression. By contrast, it has previously been reported that higher levels of WISP-1 were expressed in $46 \%$ of the patients with primary breast cancer (27). WISP-1 has also been shown to have tumour suppressive actions in H460 lung cancers (8) and in the equivalent molecule in mice, mELM1, is down-regulated in highly metastatic mouse melanoma cells (28). It is possible therefore that WISP-1 is a pro-aggressive factor, or a tumour suppressor dependent on tumour histology.

Our results for WISP-2 are in contrast to WISP-1, showing an association between lower transcript levels in the more aggressive tumours. Although no significant differences were found within the cancer group when indices of a more aggressive tumour were compared to the normal tissue a significant reduction in expression was found (Dukes $\mathrm{C}$ $\mathrm{p}=0.044$, poorly differentiated $\mathrm{p}=0.019$, TNM $3 \mathrm{p}=0.020$ and node positive disease $\mathrm{p}=0.048$ ). Our findings again confirm the initial work on WISP-2 in colorectal cancer which also found a reduction in RNA expression in tumours (5). There is evidence that there is disparity in the way WISP-2 functions in various cell types. As with colorectal cancers it has been found that in leiomyomas WISP-2 exerted an important function in maintaining the normal uterine phenotype and that loss of its actions may account, at least in part, for tumourigenesis (29). This contrasts with studies into WISP-2 in breast cancer where WISP-2 is overexpressed in the more aggressive tumours (25), but the expression is virtually undetected in normal mammary epithelial cell controls $(9,30)$. As with WISP-1, WISP-2 appears to act differently depending on the tumour type.

The results for WISP-3 suggest that the protein behaves as neither a promoter nor a tumour suppressor with human breast tumours. There were no significant differences in any of the clinically significant prognostic tests and was found in such low levels in both normal and tumour tissues that its involvement as a cancer modifier is unlikely. WISP-3 has previously been described to be overexpressed in colorectal cancers (5) and in mircosatellite instability, a phenotype of colorectal cancers with defect mismatch repair (31), but we have no significant data to substantiate these claims. By contrast, WISP-3 expression is lost in the majority of inflammatory breast cancers, a highly aggressive and metastatic form of breast cancer (32), but when it is restored the growth of inflammatory breast cancer cells in both in vitro and in vivo is inhibited, therefore, WISP-3 is acting as a tumour suppressior gene (13). It was reported that by inhibiting WISP-3 expression in human mammary epithelial cells they could induce epithelial-mesenchymal transition, promoting anchorage-independent growth, motility and invasiveness, and sensitized the cells to the growth effects of IGF-1 (14). The pathological and clinical implications of altered WISP-3 expression remains unclear and we have not demonstrated any significant association in colorectal cancer.

The clear contrast between each of the different WISPs suggests very different roles for the proteins in the development of colorectal cancer. The mechanism as to why there should be such variation is still for the most part unclear.

In conclusion, the CNN family members WISP-1, 2 and 3 display different patterns of aberration in their expression in human colorectal cancer. WISP-1 is clearly associated with an aggressive tumour linked with high levels found in the patients with the poorest prognostic outlook. In contrast WISP-2 shows some tumour suppressive activities with the transcript level being inversely proportional to tumour aggression. The WISP-3 levels show no relationship with prognostic indicators in either a protective or detrimental way. The levels of expression were extremely low and it can be concluded that as a clinical marker for disease it is of little value in colorectal cancer. The contrasting expression patterns of WISP-1 and WISP-2 may indicate a therapeutic and prognostic role which warrants further investigation.

\section{Acknowledgements}

We would like to thank the Cancer Research Wales for supporting our work.

\section{References}

1. Planque $\mathrm{N}$ and Perbal $\mathrm{B}$ : A structural approach to the role of CCN (CYR61/CTGF/NOV) proteins in tumourigenesis. Cancer Cell Int 3: 15-30, 2003.

2. Nusse R and Varmus H: Many tumors induced by the mouse mammary tumor virus contain a provirus integrated in the same region of the host genome. Cell 31: 99-109, 1982.

3. Ooyen V and Nusse R: Structure and nucleotide sequence of the putative mammary oncogene int-1; proviral insertions leave the protein-encoding domain intact. Cell 39: 233-240, 1984.

4. Tsukamoto A, Grosschedl R, Guzman R, Parslow T and Varmus H: Expression of the int-1 gene in transgenic mice is associated with mammary gland hyperplasia and adenocarcinomas in male and female mice. Cell 55: 619-625, 1988.

5. Pennica D, Swanson T, Welsh J, et al: WISP genes are members of the connective tissue growth factor family that are up-regulated in Wnt-1 transformed cells and aberrantly expressed in human colon tumours. Proc Natl Acad Sci USA 95: 14717-14722, 1998.

6. Bringstock D: The CCN family: a new stimulus package. J Endocrinol 178: 169-175, 2003.

7. Perbal B, Brigstock D and Lau L: Report on the second international workshop on the CCN family of genes. Mol Pathol 56: 80-85, 2003.

8. Khor T, Gul Y, Ithnin H and Seow H: A comparative study of the expression of Wnt-1, WISP-1, surviving and cyclin-D1 in colorectal cancer. Int J Colorectal Dis 21: 291-300, 2006.

9. Soon L, Yie T, Shvarts Levine A, Su F and Tchou-Wong K: Overexpression of WISP-1 down regulated motility and invasion of lung cancer cells through inhibition of rac activation. J Biol Chem 278: 11465-11470, 2003.

10. Su F, Overholtzer M, Besser D and Levine A: WISP-1 attenuates p53-mediated apoptosis in response to DNA damage through activation of the Akt kinase. Genes Dev16: 46-57, 2002.

11. Banerjee S, Sengupta K, Saxena N, Dhar K and Banerjee S: Epidermal growth factor induces WISP-2/CCN5 expression in estrogen receptor- $\alpha$-positive breast tumour calls through multipul modular cross-talks. Mol Cancer Res 3: 151-162, 2003. 
12. Banerjee S, Saxena N, Sengupta K, Tawfik O, Mayo M and Banerjee S: WISP-2 gene in human breast cancer: estrogen and progesterone inducible expression and regulation of tumor cell proliferation. Neoplasia 5: 63-73, 2003.

13. Thorstensen L, Diep C, Meling G, Aagesen T, Ahrens C, Rognum T and Lothe R: WNT1 inducible signaling pathway protein 3, WISP-3, a novel target gene in colorectal carcinomas with microsatellite instability. Gastroenterology 121: 1275-1280, 2001.

14. Davis L, Chen Y and Sen M: WISP-3 functions as a ligand and promotes superoxide dismutase activity. Biochem Biophys Res Commun 342: 259-265, 2006.

15. Hanavadi S, Martin T, Mansel R and Jiang WG: Interleukin-11 and its receptor expression in human breast cancer. Ann Surg Oncol 13: 802-808, 2006.

16. Martin T, Goyal A, Mansel R, Watkins G and Jiang WG: The transcription factor for the E-cadherin complex, Twist, Slug and Snail, in human breast cancer. Ann Surg Oncol 12: 1-9, 2005.

17. Jiang WG, Watkins G, Lane J, Cunnick G, Douglas-Jones A, Mokbel K and Mansel RE: Prognostic value of Rho family and rho-GDIs in breast cancer. Clin Cancer Res 9: 6432-6440, 2003.

18. Nazarenko A, Bhatnagar S and Hohman R: A closed tube format for amplification and detection of DNA based on energy transfer. Nucleic Acid Res 25: 2516-2521, 1997.

19. Jiang WG, Douglas-Jones A and Mansel RE: Level of expression of PPAR-gamma and its co-activator (PPAR-GCA) in human breast cancer. Int J Cancer 106: 752-757, 2003.

20. Ye L, Martin T, Parr C, Harrison G, Mansel R and Jiang WG: Biphasic effects of 17- $\beta$-estradiol on expression of occluding and transendothelial resistance (TER) and paracellular permeability in human vascular endothelial cells. J Cell Physiol 196: 362-369, 2003.

21. King J, Ofori-Acquash A, Stevens T, Al-Mehdi A, Fodstad O and Jiang WG: Activated leukocyte adhesion molecule in breast cancer: prognostic indicator. Breast Cancer Res 6: 478-487, 2004.

22. Parr C, Watkins G and Jiang WG: The possible correlation of Notch-1 and Notch-2 with clinical outcome and tumour clinicopathological parameters in human breast cancer. Int J Mol Med 14: 779-786, 2004
23. Jiang WG, Grimshaw D, Lane J, Martin T, Abounader R, Laterra $\mathrm{J}$ and Mansel RE: A hammerhead ribozyme suppresses expression of hepatocyte growth factor/scatter factor receptor c-MET and reduces migration and invasiveness of breast cancer cells. Clin Cancer Res 7: 2555-2562, 2001.

24. Davies G, Jiang WG and Mason M: Cell-cell adhesion and signalling intermediates in human prostate cancer. J Urol 163: 985-992, 2003.

25. Davies SR, Watkins G, Mansel RE and Jiang WG: Differential expression of the CCN family member WISP-1, WISP-2 and WISP-3 in human breast cancer and the prognostic implications. Ann Surg Oncol 14: 1909-1918, 2007.

26. Dukes CE: The surgical pathology of rectal cancer. Am J Surg 1950: 66-71, 1950 .

27. Xie D, Nakachi K, Wang H, Elashoff R and Koeffler P: Elevated levels of connective tissue growth factor, WISP-1 and CYR61 in primary breast cancers associated with more advanced features. Cancer Res 61: 8917-8923, 2001.

28. Babic A, Kireeva M, Kolesnikova T and Lau L: CYR61, a product of a growth factor-inducible immediate early gene, promotes angiogenesis and tumor growth. Proc Natl Acad Sci USA 95: 6355-6360, 1998.

29. Mason H, Lake A, Wubben J, Nowak R and Castellot J Jr: The growth arrest-specific gene CCN5 is deficient in human leiomyomas and inhibits the proliferation and motility of cultured human uterine smooth muscle cells. Mol Hum Reprod 10: 181-187, 2004.

30. Zoubine M, Banerjee S, Saxena N, Campbell D and Banerjee S: WISP-2: a serum-induible gene differentially expressed in human normal breast epithelial cells and in MCF-7 breast tumor cells. Biochem Biophys Res Commun 282: 421-425, 2001.

31. Thorstenesen L, Diep CB, Meling GI, Aagesen TH, Ahrens CH, Rognum TO and Lothe RA: WNT1 inducible signalling pathway protein 3, WISP-3, a novel target gene in colorectal carcinomas with microsatellite instability. Gastroenterology 121: 1275-1280, 2001

32. Van Golen K, Davies S, Wu Z, et al: A novel putative lowaffinity insulin-like growth factor-binding protein, LIBC (lost in inflammatory breast cancer), and RhoC GTPase correlate with the inflammatory breast cancer phenotype. Clin Cancer Res 5: 2511-2519, 1999. 